\title{
Construction of spline curves on smooth manifolds by action of Lie groups
}

\author{
A. P. Pobegailo
}

\section{Abstract}

Polynomials for blending parametric curves in Lie groups are defined. Properties of these polynomials are proved. Blending parametric curves in Lie groups with these polynomials is considered. Then application of the proposed technique to construction of spline curves on smooth manifolds is presented. As an example, construction of spherical spline curves using the proposed approach is depicted.

\section{Introduction}

This article deals with the problem of blending parametric curves in Lie groups and application of the blending technique to construction of spline curves on smooth manifolds. The different known approaches to construction of spline curves on smooth manifolds and in Lie groups are mainly based on two techniques. The first technique is that spline curves are constructed in a linear space provided that the curves belong to the smooth manifold which is embedded in the linear space. In this case some optimization methods are usually used for fitting the designed curve on the smooth manifold $[\mathbf{1}, \mathbf{3}-\mathbf{5}, \mathbf{8}, \mathbf{1 0}]$. The second approach uses geodesics for construction of spline curves on smooth manifolds and Lie groups $[\mathbf{2}, \mathbf{7}, \mathbf{9}, \mathbf{1 2}]$. These two techniques usually use sophisticated numerical methods to compute the necessary spline curves on manifolds or need extensive computations concerned with the exponential mapping. Therefore the approaches can be used only for off-line applications concerned with construction of spline curves on smooth manifolds. The approach to construction of spline curves on smooth manifolds by action of Lie groups was introduced in [11]. The same approach is also presented in [6] but using the exponential mapping. The paper [13] presents a geometric approach to interpolating spline curves on Riemannian manifolds which combines the first approach and the method considered in [6].

The article presents an approach to blending of parametric curves in Lie groups and construction of spline curves on smooth manifolds by means of blending one-parameter subgroups acting on the smooth manifold. For blending parametric curves in Lie groups two classes of polynomials which satisfy specific boundary conditions are introduced. The boundary conditions of these polynomials ensure the necessary smoothness of a spline curve on a smooth manifold. The presented approach can be considered as an improvement of the method proposed in the article [11]. There are two reasons for this improvement. Firstly, the proposed polynomials have extremal energy properties which ensure optimal transition between blended parametric curves. Secondly, smoothing polynomials have lower degrees which implies faster on-line computations of blending parametric curves in Lie groups, especially when parameter values of the parametric curve are adjusted. The presented approach is aimed at geometric applications concerned with on-line interpolation on Lie groups and smooth manifolds because it is computationally simple and the spline curves are constructed locally.

Received 1 June 2014; revised 29 September 2014.

2010 Mathematics Subject Classification 65D05 (primary), 65D07, 65D17 (secondary). 


\section{The problem of blending parametric curves in Lie groups}

Formally the problem of blending parametric curves in Lie groups can be stated as follows. Let $g_{1}(u)$ and $g_{2}(u), u \in[0,1]$, be two arbitrary parametric curves which belong to a Lie group $G$ and satisfy the initial conditions

$$
g_{1}(0)=g_{2}(0)=e
$$

where $e$ denotes a unity of the Lie group $G$. The problem is to construct a parametric curve $g(u) \in G, u \in[0,1]$, that satisfies the boundary conditions

$$
\begin{gathered}
g(0)=g_{2}(0)=e, \quad g(1)=g_{1}(1) \\
g^{(m)}(0)=g_{2}^{(m)}(0), \quad g^{(m)}(1)=g_{1}^{(m)}(1), \quad \text { for all } m \in\{1,2, \ldots, n\}
\end{gathered}
$$

where $n \in N$. A parametric curve $g(u)$ which satisfies the conditions is called a parametric curve blending the parametric curves $g_{1}(u)$ and $g_{2}(u)$. The parametric curve $g(u)$ can also be considered as a smooth transition from the parametric curve $g_{2}(u)$ to the parametric curve $g_{1}(u)$. It should be noted that in geometric applications $g_{1}(u)$ and $g_{2}(u)$ are usually one-parameter subgroups of the matrix Lie group $G$. This observation clarifies the problem statement. A description of Lie groups and smooth manifolds can be found in [15].

Taking into consideration that $G$ is a Lie group, suppose that a solution of the problem is a parametric curve of the form

$$
g(u)=g_{1}\left(f_{1, n}(u)\right) g_{2}\left(f_{2, n}(u)\right), \quad u \in[0,1],
$$

where $f_{1, n}(u)$ and $f_{2, n}(u)$ are some real functions which provide fulfillment of the conditions defined by equalities (2.2), (2.3) and have some extremal properties. Therefore the problem now is to determine the desired real functions $f_{1, n}(u)$ and $f_{2, n}(u)$. In the next sections, two classes of polynomial functions $w_{1, n}(u)$ and $w_{2, n}(u)$, which solve the problem, are introduced and extremal properties of the polynomials are proved. Then an application of the presented technique to construction of spline curves on smooth manifolds is considered.

\section{Smoothly increasing polynomials}

We introduce the first class of polynomials which will be used for blending parametric curves in Lie groups. To construct the polynomials consider the following sequences of knot points on the real line $R$ :

$$
(\underbrace{0,0, \ldots, 0}_{n+1}, \frac{n+1}{2 n+1}, \frac{n+2}{2 n+1}, \ldots, \frac{2 n+1}{2 n+1}), \quad n \in N .
$$

Using Bernstein polynomials

$$
b_{n, m}(u)=\left(\begin{array}{c}
n \\
m
\end{array}\right)(1-u)^{n-m} u^{m}, \quad u \in[0,1],
$$

define the following Bézier curves on the sequences of knot points:

$$
w_{1, n}(u)=\sum_{i=1}^{n+1} b_{2 n+1, n+i}(u) \frac{n+i}{2 n+1}, \quad n \in N .
$$

It follows from this definition that the polynomials $w_{1, n}(u)$ satisfy the boundary conditions

$$
w_{1, n}(0)=0, \quad w_{1, n}(1)=1 .
$$


Derivatives of the polynomials $w_{1, n}(u)$ at the boundaries can be computed using expressions for derivatives of Bézier curves at boundary points [14] as follows:

$$
\begin{gathered}
w_{1, n}^{(m)}(0)=0, \quad \text { for all } m \in\{1,2, \ldots, n\} \\
w_{1, n}^{(m)}(1)=\frac{(2 n+1) !}{(2 n+1-m) !} \sum_{i=0}^{m}(-1)^{i}\left(\begin{array}{c}
m \\
i
\end{array}\right) \frac{2 n+1-i}{2 n+1}, \quad \text { for all } m \in\{1,2, \ldots, n\} .
\end{gathered}
$$

Determine values of the derivatives which are defined by equality (3.4). A derivative of the first order can be computed as follows:

$$
w_{1, n}^{\prime}(1)=\frac{(2 n+1) !}{(2 n) !}\left(\frac{2 n+1}{2 n+1}-\frac{2 n}{2 n+1}\right)=\frac{(2 n) !}{(2 n) !}=1 .
$$

Using identities with binomial coefficients, which are considered in the appendix, higher-order derivatives of the polynomials $w_{1, n}(u)$ can be computed as follows:

$$
\begin{aligned}
w_{1, n}^{(m)}(1) & =\frac{(2 n+1) !}{(2 n+1-m) !} \sum_{i=0}^{m}(-1)^{i}\left(\begin{array}{c}
m \\
i
\end{array}\right) \frac{2 n+1-i}{2 n+1} \\
& =\frac{(2 n+1) !}{(2 n+1-m) !}\left(\sum_{i=0}^{m}(-1)^{i}\left(\begin{array}{c}
m \\
i
\end{array}\right)-\frac{1}{2 n+1} \sum_{i=0}^{m}(-1)^{i}\left(\begin{array}{c}
m \\
i
\end{array}\right) i\right)=0
\end{aligned}
$$

for all $m \in\{2,3, \ldots, n\}$. Therefore the derivatives of the polynomials $w_{1, n}(u)$ defined by equality (3.4) have the following values:

$$
w_{1, n}^{\prime}(1)=1, \quad w_{1, n}^{(m)}(1)=0, \quad \text { for all } m \in\{2,3, \ldots, n\} .
$$

The following polynomials of lower degrees

$$
\begin{gathered}
w_{1,1}(u)=2(1-u) u^{2}+u^{3}, \\
w_{1,2}(u)=6(1-u)^{2} u^{3}+4(1-u) u^{4}+u^{5}
\end{gathered}
$$

are usually used in geometric applications.

Figure 1 shows graphs of the polynomials $w_{1, n}(u)$. This figure illustrates kinematics features of these polynomials. It can be seen that the polynomials $w_{1, n}(u)$ can be considered as rather good approximations of the linear function which has a unity motion speed. In other words the polynomials $w_{1, n}(u)$ smoothly increase motion speed from zero to a unity on the interval $[0,1]$ with the necessary continuity at the boundaries of the interval.

We show that the polynomial $w_{1, n}(u)$ is a minimum of the functional

$$
J_{n}(f)=\int_{0}^{1}\left|f^{(n+1)}(u)\right|^{2} d u, \quad n \in N,
$$

where the function $f(u), u \in[0,1]$, satisfies the boundary conditions

$$
\begin{gathered}
f(0)=0, \quad f(1)=1, \\
f^{(m)}(0)=0, \quad \text { for all } m \in\{1,2, \ldots, n\} \\
f^{\prime}(1)=1, \quad f^{(m)}(1)=0, \quad \text { for all } m \in\{2,3, \ldots, n\} .
\end{gathered}
$$

Assume that a function $g(u)$ is a minimum of the functional $J_{n}(f)$. Consider the function

$$
\left(g-w_{1, n}\right)(u)=g(u)-w_{1, n}(u) .
$$




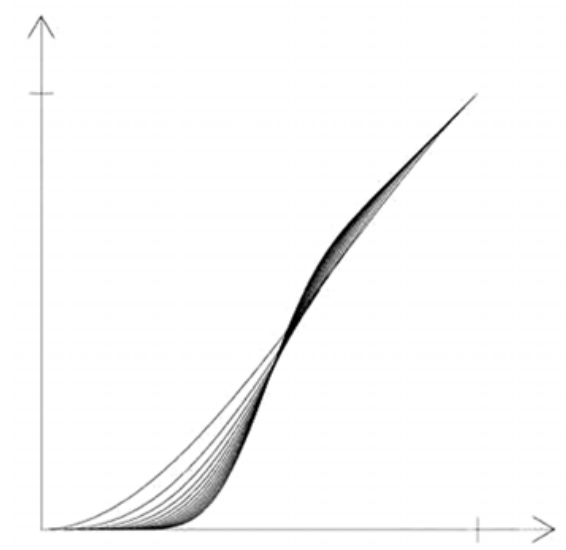

FiguRE 1. Graphs of the polynomials $w_{1, n}(u)$.

Then

$$
\begin{aligned}
\left|\left(g-w_{1, n}\right)^{(n)}\right|^{2} & =\left|g^{(n)}-w_{1, n}^{(n)}\right|^{2}=\left(g^{(n)}\right)^{2}-2 g^{(n)} w_{1, n}^{(n)}+\left(w_{1, n}^{(n)}\right)^{2} \\
& =\left(g^{(n)}\right)^{2}-\left(w_{1, n}^{(n)}\right)^{2}-2\left(g^{(n)}-w_{1, n}^{(n)}\right) w_{1, n}^{(n)} .
\end{aligned}
$$

It follows from these equations that

$$
J_{n}\left(g-w_{1, n}\right)=J_{n}(g)-J_{n}\left(w_{1, n}\right)-2 \int_{0}^{1}\left(g^{(n+1)}(u)-w_{1, n}^{(n+1)}(u)\right) w_{1, n}^{(n+1)}(u) d u
$$

The last integral can be computed by parts as follows:

$$
\begin{aligned}
& \int_{0}^{1}\left(g^{(n+1)}(u)-w_{1, n}^{(n+1)}(u)\right) w_{1, n}^{(n+1)}(u) d u=\int_{0}^{1} w_{1, n}^{(n+1)}(u) d\left(g^{(n)}(u)-w_{1, n}^{(n)}(u)\right) \\
& \quad=\left.w_{1, n}^{(n+1)}(u)\left(g^{(n)}(u)-w_{1, n}^{(n)}(u)\right)\right|_{0} ^{1}-\int_{0}^{1}\left(g^{(n)}(u)-w_{1, n}^{(n)}(u)\right) w_{1, n}^{(n+2)}(u) d u \\
& \quad=-\int_{0}^{1}\left(g^{(n)}(u)-w_{1, n}^{(n)}(u)\right) w_{1, n}^{(n+2)}(u) d u
\end{aligned}
$$

taking into account that

$$
g^{(n)}(0)=w_{1, n}^{(n)}(0)=0, \quad g^{(n)}(1)=w_{1, n}^{(n)}(1)=0
$$

according to equalities (3.7) and (3.8) respectively. Computing the obtained integrals by parts recurrently and taking into account that the function $w_{1, n}^{(2 n+1)}(u)$ is a constant it is obtained that

$$
\begin{aligned}
\int_{0}^{1}\left(g^{(n+1)}(u)-w_{1, n}^{(n+1)}(u)\right) w_{1, n}^{(n+1)}(u) d u & =(-1)^{n} \int_{0}^{1}\left(g^{\prime}(u)-w_{1, n}^{\prime}(u)\right) w_{1, n}^{(2 n+1)}(u) d u \\
& =\left.(-1)^{n}\left(g(u)-w_{1, n}(u)\right) w_{1, n}^{(2 n+1)}(u)\right|_{0} ^{1}=0
\end{aligned}
$$


taking into account that

$$
g(0)=w_{1, n}(0)=0, \quad g(1)=w_{1, n}(1)=1
$$

according to equalities (3.6). Thus it is proved that

$$
J_{n}\left(g-w_{1, n}\right)=J_{n}(g)-J_{n}\left(w_{1, n}\right) .
$$

The last equation can be rewritten as

$$
J_{n}(g)=J_{n}\left(w_{1, n}\right)+J_{n}\left(g-w_{1, n}\right) .
$$

It follows from the definition of the functional $J_{n}(f)$ that

$$
J_{n}\left(g-w_{1, n}\right) \geqslant 0 .
$$

Therefore

$$
J_{n}\left(w_{1, n}\right) \leqslant J_{n}(g) .
$$

But the function $g(u)$ is a minimum of the functional $J_{n}(f)$ by assumption, therefore

$$
g(u)=w_{1, n}(u) .
$$

Thus it is proved that the polynomial $w_{1, n}(u)$ is a minimum of the functional $J_{n}(f)$.

Now we prove that this minimum is unique. Suppose the opposite. Let there exist a function $g(u)$ which satisfies the condition

$$
J_{n}(g)=J_{n}\left(w_{1, n}\right)
$$

It follows from this equation that

$$
J_{n}\left(g-w_{1, n}\right)=0
$$

or equivalently

$$
g^{(n+1)}(u)=w_{1, n}^{(n+1)}(u), \quad \text { for all } u \in[0,1] .
$$

It follows from the last equation that

$$
g(u)=w_{1, n}(u)+\sum_{i=0}^{n} a_{i} u^{i} .
$$

But the coefficients $a_{i}$ are equal to zero for all $i \in\{0,1, \ldots, n\}$ taking into account boundary conditions which must be satisfied by the function $g(u)$. Therefore

$$
g(u)=w_{1, n}(u)
$$

Thus the property is proved. The functional $J_{n}(f)$ can be considered as energy of the $(n+1)$ th derivative of the function which satisfies boundary conditions defined by equalities (3.6)-(3.8).

\section{Smoothly decreasing polynomials}

We introduce the second class of polynomials which will be used for blending parametric curves in Lie groups. To construct the polynomials consider the following sequences of knot points on the real line $R$ :

$$
(0, \frac{1}{2 n+1}, \frac{2}{2 n+1}, \ldots, \frac{n}{2 n+1}, \underbrace{0,0, \ldots, 0}_{n+1}), \quad n \in N .
$$


Using Bernstein polynomials $b_{n, m}(u), u \in[0,1]$, define the following Bézier curves on the sequences of knot points:

$$
w_{2, n}(u)=\sum_{i=1}^{n} b_{2 n+1, i}(u) \frac{i}{2 n+1}, \quad n \in N
$$

It follows from this definition that the polynomials $w_{2, n}(u)$ satisfy the boundary conditions

$$
w_{2, n}(0)=0, \quad w_{2, n}(1)=0 .
$$

Derivatives of the polynomials $w_{2, n}(u)$ at the boundaries can be computed using expressions for derivatives of Bézier curves at boundary points [14] as follows:

$$
\begin{gathered}
w_{2, n}^{(m)}(0)=\frac{(2 n+1) !}{(2 n+1-m) !} \sum_{i=0}^{m}(-1)^{m-i}\left(\begin{array}{c}
m \\
i
\end{array}\right) \frac{i}{2 n+1}, \quad \text { for all } m \in\{1,2, \ldots, n\} \\
w_{2, n}^{(m)}(1)=0, \quad \text { for all } m \in\{1,2, \ldots, n\} .
\end{gathered}
$$

We determine values of the derivatives which are defined by equality (4.3). A derivative of the first order can be computed as follows:

$$
w_{2, n}^{\prime}(0)=\frac{(2 n+1) !}{(2 n) !} \frac{1}{2 n+1}=\frac{(2 n) !}{(2 n) !}=1 .
$$

Using identities with binomial coefficients, which are considered in the appendix, higher-order derivatives of the polynomials $w_{2, n}(u)$ can be computed as follows:

$$
\begin{aligned}
w_{2, n}^{(m)}(1) & =\frac{(2 n+1) !}{(2 n+1-m) !} \sum_{i=0}^{m}(-1)^{m-i}\left(\begin{array}{c}
m \\
i
\end{array}\right) \frac{i}{2 n+1} \\
& =\frac{(2 n+1) !}{(2 n+1-m) !} \frac{(-1)^{m}}{2 n+1} \sum_{i=0}^{m}(-1)^{i}\left(\begin{array}{c}
m \\
i
\end{array}\right) i=0
\end{aligned}
$$

for all $m \in\{2,3, \ldots, n\}$. Therefore the derivatives of the polynomials $w_{2, n}(u)$ defined by equality (4.3) have the following values:

$$
w_{2, n}^{\prime}(0)=1, \quad w_{2, n}^{(m)}(0)=0, \quad \text { for all } m \in\{2,3, \ldots, n\} .
$$

The polynomials of lower degrees,

$$
\begin{gathered}
w_{2,1}(u)=(1-u)^{2} u, \\
w_{2,2}(u)=(1-u)^{2} u^{3}+4(1-u) u^{4},
\end{gathered}
$$

are usually used in geometric applications.

Figure 2 shows graphs of the polynomials $w_{2, n}(u)$. This figure illustrates kinematics features of these polynomials. It can be seen that the polynomials $w_{2, n}(u)$ smoothly decrease motion speed from a unity to zero on the interval $[0,1]$ with the necessary continuity at the boundaries of the interval.

The polynomial $w_{2, n}(u)$ is a minimum of the functional

$$
J_{n}(f)=\int_{0}^{1}\left|f^{(n+1)}(u)\right|^{2} d u, \quad n \in N
$$




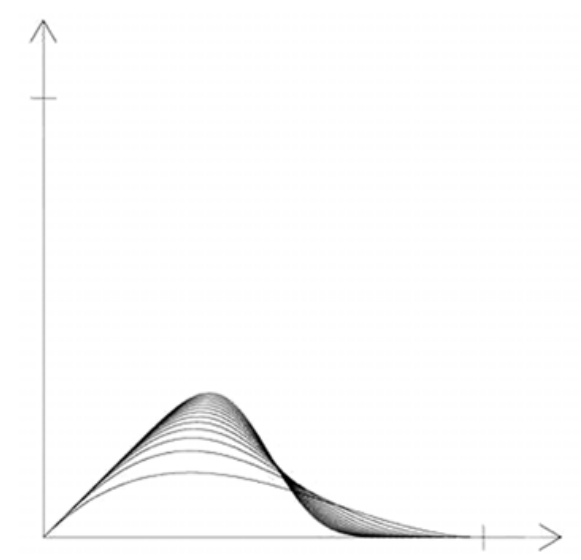

Figure 2. Graphs of the polynomials $w_{2, n}(u)$.

where the function $f(u), u \in[0,1]$, satisfies the boundary conditions

$$
\begin{gathered}
f(0)=0, \quad f(1)=0, \\
f^{\prime}(0)=1, \quad f^{(m)}(0)=0, \quad \text { for all } m \in\{2,3, \ldots, n\} \\
f^{(m)}(1)=0, \quad \text { for all } m \in\{1,2, \ldots, n\}
\end{gathered}
$$

This property can be proved by analogy with the corresponding property of the polynomials $w_{1, n}(u)$ which was considered in $\S 3$.

\section{Blending parametric curves in matrix Lie groups}

Now we solve the problem of blending parametric curves in Lie groups which was stated in $\S 2$. For this purpose consider two arbitrary parametric curves $g_{1}(u)$ and $g_{2}(u), u \in[0,1]$, which belong to a matrix Lie group $G$ and satisfy the initial conditions defined by equalities (2.1). It should be noted that since any point $g \in G$ can be chosen as a unity of the group $G$ by transition to another coordinate system, use of $e$ as the initial point is not important. The problem is to construct a parametric curve $g(u) \in G, u \in[0,1]$, that satisfies the boundary conditions defined by equalities (2.2) and (2.3).

In order to solve the problem construct a parametric curve $g(u)$ using polynomials $w_{1, n}(u)$ and $w_{2, n}(u)$ defined by equalities (3.1) and (4.1) as follows:

$$
g(u)=g_{1}\left(w_{1, n}(u)\right) g_{2}\left(w_{2, n}(u)\right), \quad u \in[0,1]
$$

taking into account equality (2.4). Show that the parametric curve $g(u)$ satisfies the boundary conditions defined by equalities (2.2) and (2.3).

It follows from the initial conditions defined by equalities (2.1) that

$$
g(0)=g_{1}\left(w_{1, n}(0)\right) g_{2}\left(w_{2, n}(0)\right)=g_{1}(0) g_{1}(0)=e \circ e=e
$$

and

$$
g(1)=g_{1}\left(w_{1, n}(1)\right) g_{2}\left(w_{2, n}(1)\right)=g_{1}(1) g_{2}(0)=g_{1}(1) \circ e=g_{1}(1)
$$


taking into account that the polynomials $w_{1, n}(u)$ and $w_{2, n}(u)$ satisfy the boundary conditions defined by equalities (3.2) and (4.2) respectively. Thus the equalities (2.2) are fulfilled.

Now we prove the equalities (2.3). For this purpose firstly compute higher-order derivatives of parametric curves $g_{1}\left(w_{2, n}(u)\right)$ and $g_{2}\left(w_{1, n}(u)\right)$ at the boundaries using the expression for higher-order derivatives of a composite function considered in the appendix. It is obtained using equalities (4.2), (4.4) and (4.5) that

$$
\begin{aligned}
& \left(g_{2}\left(w_{2, n}(u)\right)\right)^{(m)}(0) \\
& \quad=\left(\sum \frac{m !}{k_{1} ! k_{2} ! \ldots k_{m} !} g_{2}^{(k)}\left(w_{2, n}(u)\right)\left(\frac{w_{2, n}^{\prime}(u)}{1 !}\right)^{k_{1}}\left(\frac{w_{2, n}^{\prime \prime}(u)}{2 !}\right)^{k_{2}} \ldots\left(\frac{w_{2, n}^{(m)}(u)}{m !}\right)^{k_{m}}\right)(0) \\
& \quad=\sum \frac{m !}{k_{1} ! k_{2} ! \ldots k_{m} !} g_{2}^{(k)}(0)\left(\frac{w_{2, n}^{\prime}(0)}{1 !}\right)^{k_{1}}\left(\frac{w_{2, n}^{\prime \prime}(0)}{2 !}\right)^{k_{2}} \ldots\left(\frac{w_{2, n}^{(m)}(0)}{m !}\right)^{k_{m}} \\
& \quad=g_{2}^{(m)}(0)\left(w_{2, n}^{\prime}(0)\right)^{m}=g_{2}^{(m)}(0)
\end{aligned}
$$

and

$$
\begin{aligned}
& \left(g_{2}\left(w_{2, n}(u)\right)\right)^{(m)}(1) \\
& \quad=\left(\sum \frac{m !}{k_{1} ! k_{2} ! \ldots k_{m} !} g_{2}^{(k)}\left(w_{2, n}(u)\right)\left(\frac{w_{2, n}^{\prime}(u)}{1 !}\right)^{k_{1}}\left(\frac{w_{2, n}^{\prime \prime}(u)}{2 !}\right)^{k_{2}} \ldots\left(\frac{w_{2, n}^{(m)}(u)}{m !}\right)^{k_{m}}\right) \\
& \quad=\sum \frac{m !}{k_{1} ! k_{2} ! \ldots k_{m} !} g_{2}^{(k)}(1)\left(\frac{w_{2, n}^{\prime}(1)}{1 !}\right)^{k_{1}}\left(\frac{w_{2, n}^{\prime \prime}(1)}{2 !}\right)^{k_{2}} \ldots\left(\frac{w_{2, n}^{(m)}(1)}{m !}\right)^{k_{m}}=0
\end{aligned}
$$

for all $m \in\{1,2, \ldots, n\}$. Analogously it can be obtained using equalities (3.2), (3.3) and (3.5) that

$$
\left(g_{1}\left(w_{1, n}(u)\right)\right)^{(m)}(0)=0, \quad\left(g_{1}\left(w_{1, n}(u)\right)\right)^{(m)}(1)=g_{1}^{(m)}(1), \quad \text { for all } m \in\{1,2, \ldots, n\} .
$$

Now compute higher-order derivatives of the parametric curve $g(u)$ at its boundaries. It is obtained taking into account the computed higher-order derivative values of the parametric curves $g_{1}\left(w_{2, n}(u)\right)$ and $g_{2}\left(w_{1, n}(u)\right)$ that

$$
\begin{aligned}
g^{(m)}(0) & =\left(g_{1}\left(w_{1, n}(u)\right) g_{2}\left(w_{2, n}(u)\right)\right)^{(m)}(0) \\
& =\sum_{k=0}^{m}\left(\begin{array}{c}
m \\
k
\end{array}\right)\left(g_{1}\left(w_{1, n}(u)\right)\right)^{(k)}(0)\left(g_{2}\left(w_{2, n}(u)\right)\right)^{(m-k)}(0) \\
& =g_{1}\left(w_{1, n}(u)\right)(0)\left(g_{2}\left(w_{2, n}(u)\right)\right)^{(m)}(0)=g_{1}(0) g_{2}^{(m)}(0) \\
& =e \circ g_{2}^{(m)}(0)=g_{2}^{(m)}(0)
\end{aligned}
$$

and analogously

$$
g^{(m)}(1)=g_{1}^{(m)}(1)
$$

for all $m \in\{1,2, \ldots, n\}$. Thus equalities (2.3) are also proved.

In this section, for simplicity, only matrix Lie groups were considered. But taking into account that any Lie group is locally homeomorphic to a matrix Lie group through exponential mapping the obtained results can be expanded on abstract Lie groups. Therefore the presented approach can be also used for construction of blending parametric curves $g(u)$, which satisfies the conditions defined by equalities (2.1)-(2.3) on abstract Lie groups. But this is not important in geometric applications where only matrix transformations are used. 


\section{Blending parametric curves on smooth manifolds}

Consider a smooth manifold $M$ and let $G$ be a Lie group acting on $M$. Choose two arbitrary different points $p_{1}$ and $p_{2}$ which lie on the smooth manifold $M$ and suppose that the points are connected by two different smooth parametric curves $q_{1}(u)$ and $q_{2}(u)$ which are defined as follows:

$$
q_{j}(u)=g_{j}(u) p_{1}, \quad u \in[0,1], j \in\{1,2\}
$$

where $g_{1}(u)$ and $g_{2}(u)$ are some parametric curves in the Lie group $G$ which satisfy the initial conditions defined by equality (2.1). It follows from this definition that

$$
q_{j}(0)=g_{j}(0) p_{1}=p_{1}, \quad q_{j}(1)=g_{j}(1) p_{1}=p_{2}, j \in\{1,2\} .
$$

The problem is to construct a parametric curve $q(u) \in M$ which satisfies the following boundary conditions:

$$
\begin{gathered}
q(0)=p_{1}, \quad q(1)=p_{2}, \\
q^{(m)}(0)=q_{2}^{(m)}(0), \quad q^{(m)}(1)=q_{1}^{(m)}(1), \quad m \in\{1,2, \ldots, n\}
\end{gathered}
$$

where $n \in N$. In order to solve the problem define the parametric curve $q(u)$ as follows:

$$
q(u)=g_{1}\left(w_{1, n}(u)\right) g_{2}\left(w_{2, n}(u)\right) p_{1}=g(u) p_{1}, \quad u \in[0,1],
$$

taking into account equality (5.1). It is obvious that the parametric curve $q(u)$ belongs to the manifold $M$ because the parametric curve $g(u)$ lies in the Lie group $G$. In $\S 5$ it was proved that the parametric curve $g(u)$ satisfies equalities (2.2) and (2.3). Show that the parametric curve $q(u)$ satisfies the boundary conditions defined by equalities (6.2) and (6.3).

It follows from equalities (2.2) that

$$
\begin{aligned}
& q(0)=g(0) p_{1}=g_{2}(0) p_{1}=p_{1}, \\
& q(1)=g(1) p_{1}=g_{1}(1) p_{1}=p_{2} .
\end{aligned}
$$

Thus equalities (6.2) are fulfilled, that is, the parametric curve $q(u)$ connects the points $p_{1}$ and $p_{2}$.

Now we prove equalities (6.3). Taking into account that the parametric curve $g(u)$ satisfies equalities (2.3) it is obtained that

$$
\begin{gathered}
q^{(m)}(0)=g^{(m)}(0) p_{1}=g_{2}^{(m)}(0) p_{1}=q_{2}^{(m)}(0), \\
q^{(m)}(1)=g^{(m)}(1) p_{1}=g_{1}^{(m)}(1) p_{1}=q_{1}^{(m)}(1)
\end{gathered}
$$

for any $m \in\{1,2, \ldots, n\}$. Thus equalities (6.3) are also proved.

Since the parametric curves $q_{1}(u)$ and $q_{2}(u)$ differ only in the parametric curves $g_{1}(u)$ and $g_{2}(u)$ which are blended, the parametric curve $q(u)$ can be considered as a parametric curve defined by blending the parametric curves $q_{1}(u)$ and $q_{2}(u)$.

\section{Construction of spline curves on smooth manifolds}

In this section a general scheme for construction of spline curves on smooth manifolds by acting Lie groups is considered. A segment of the spline curve is constructed by blending two parametric curves of the Lie group acting on the smooth manifold. It can be supposed that the blending parametric curves are one-parameter subgroups of the Lie group. This is reasonable because one-parameter subgroups are geodesics and can be considered as the simplest parametric curves on Lie groups. 


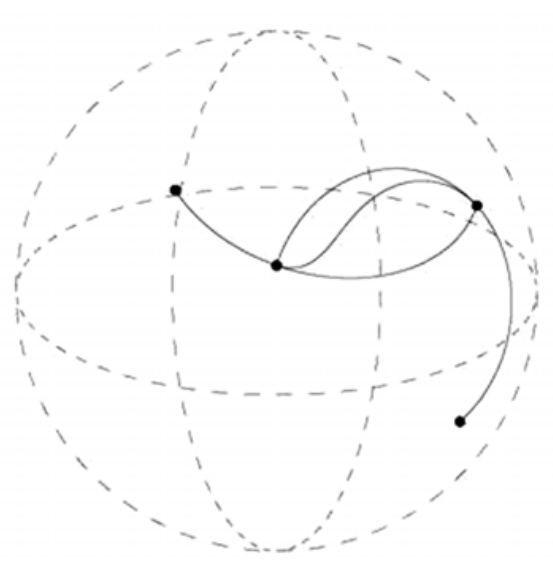

Figure 3. Spline curve segment on the sphere $S^{2}$.

Consider a sequence of points $\left(p_{i}\right)_{i=1}^{k}$ which lie on a smooth manifold $M$ and a Lie group $G$ acting on $M$. The problem is to construct a $C^{n}$ continuous spline curve $\left(q_{i}(u)\right)_{i=1}^{k-1}, u \in[0,1]$, on the smooth manifold $M$ which interpolates the points $p_{i}$. Using equality (6.4), a segment $q_{i}(u)$ of the spline curve can be constructed by blending two parametric curves

$$
q_{i, j}(u)=g_{i, j}(u) p_{i}, \quad u \in[0,1], j \in\{1,2\},
$$

which lie on the smooth manifold $M$ and connect the points $p_{i}$ and $p_{i+1}$, as follows:

$$
q_{i}(u)=g_{i, 1}\left(w_{1, n}(u)\right) g_{i, 2}\left(w_{2, n}(u)\right) p_{i}=g_{i}(u) p_{i}, \quad u \in[0,1],
$$

for all $i \in\{2,3, \ldots, k-2\}$. In accordance with the proposed approach the initial and final segments can be defined by

$$
\begin{aligned}
q_{1}(u)=q_{1,1}(u) & =g_{1,1}(u) p_{1}, \quad u \in[0,1], \\
q_{k-1}(u)=q_{k-1,2}(u) & =g_{k-1,2}(u) p_{k-1}, \quad u \in[0,1] .
\end{aligned}
$$

For example, if a spline curve segment $q_{i}(u)$ is constructed on the sphere $S^{2}$ then the parametric curves $q_{i, 1}(u)$ and $q_{i, 2}(u)$ are small circular arcs obtained by actions of some orthogonal rotations $g_{i, 1}(u)$ and $g_{i, 2}(u)$ on the point $p_{i}$. Figure 3 illustrates the example.

In order to ensure $C^{n}$ parametric continuity of the spline curve $\left(q_{i}(u)\right)_{i=1}^{k-1}$ at the knot points $p_{i}, i \in\{2,3, \ldots, k-1\}$, the parametric curves $q_{i, 1}(u)$ and $q_{i+1,2}(u)$, which are used for construction of the segments $q_{i}(u)$ and $q_{i+1}(u)$ respectively, must be at least $C^{n}$ continuously joined at the knot point $p_{i+1}$, that is

$$
q_{i, 1}^{(m)}(1)=q_{i+1,2}^{(m)}(0), \quad m \in\{1,2, \ldots, n\} .
$$

Usually it is difficult to meet the last requirement provided that the parametric curves $q_{i, 1}(u)$ and $q_{i+1,2}(u)$ have a normal parametrization. In order to avoid this difficulty some non-normal parametrization of the parametric curves can be chosen. That is, some knot parameter values $t_{i}$ are assigned to each knot point $p_{i}, i \in\{1,2, \ldots, k\}$ and the parameterizations

$$
u(t)=\frac{t-t_{i}}{t_{i+1}-t_{i}}, \quad t \in\left[t_{i}, t_{i+1}\right]
$$




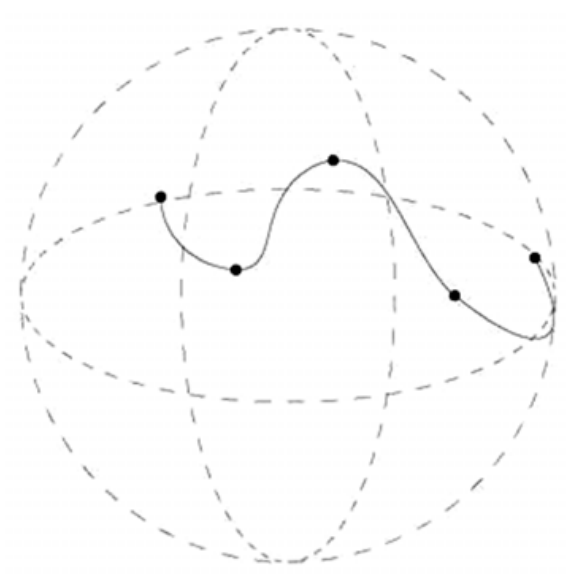

Figure 4. $C^{2}$ continuous spline curve on the sphere $S^{2}$.

are used for parametric curves $q_{i, 1}(u)$ and $q_{i, 2}(u)$. The parameter values $t_{i}$ should be chosen in such a way that the continuity conditions

$$
q_{i, 1}^{(m)}\left(t_{i+1}\right)=q_{i+1,2}^{(m)}\left(t_{i+1}\right), \quad m \in\{1,2, \ldots, n\}
$$

are fulfilled.

Suppose that the parametric curves $q_{i, 1}(u)$ and $q_{i+1,2}(u)$ are defined as follows:

$$
q_{i, 1}(u)=g_{i, 1}\left(u \varphi_{i, 1}\right) p_{i}, \quad u \in[0,1], i \in\{1,2, \ldots, k-2\},
$$

and

$$
q_{i+1,2}(u)=g_{i+1,2}\left(u \varphi_{i+1,2}\right) p_{i+1}, \quad u \in[0,1], i \in\{2,3, \ldots, k-1\},
$$

where $g_{i, 1}\left(u \varphi_{i, 1}\right)$ and $g_{i+1,2}\left(u \varphi_{i+1,2}\right)$ are parametric curves which belong to the same oneparameter subgroup of the Lie group $G$. For example, if the smooth manifold is the sphere $S^{2}$, then the parametric curves $g_{i, 1}\left(u \varphi_{i, 1}\right)$ and $g_{i+1,2}\left(u \varphi_{i+1,2}\right)$ describe orthogonal rotations about the same axis but with different angles $\varphi_{i, 1}$ and $\varphi_{i+1,2}$ respectively. Then in order to ensure $C^{n}$ parametric continuity of the spline curve, the parameter values $t_{i}$ can be defined as follows:

$$
\begin{gathered}
t_{1}=0, \quad t_{2}=\varphi_{1,1} \\
t_{i+1}=t_{i}+\frac{\varphi_{i+1,2}}{\varphi_{i, 1}}\left(t_{i}-t_{i-1}\right), \quad i \in\{2,3, \ldots, k-1\} .
\end{gathered}
$$

It can be seen that in this case

$$
\left(\frac{t-t_{i}}{t_{i+1}-t_{i}}\right)^{\prime} \varphi_{i+1,2}=\frac{\varphi_{i+1,2}}{t_{i+1}-t_{i}}=\frac{\varphi_{i, 1}}{t_{i}-t_{i-1}}=\left(\frac{t-t_{i-1}}{t_{i}-t_{i-1}}\right)^{\prime} \varphi_{i, 1}
$$

and therefore the parametric curves $q_{i, 1}(u(t))$ and $q_{i+1,2}(u(t))$ are $C^{n}$ continuously joined at the knot point $p_{i+1}$. Then it follows from equality $(6.3)$ that the constructed spline curve $\left(q_{i}(t)\right)_{i=1}^{k-1}, t \in\left[t_{1}, t_{k}\right]$, is $C^{n}$ continuous.

Figure 4 shows the $C^{2}$ continuous spline curve constructed by the proposed approach on the sphere $S^{2}$. 


\section{Conclusions}

Polynomials for blending parametric curves in Lie groups are introduced. Extremal properties of these polynomials are proved. The technique of blending parametric curves in Lie groups with these polynomials is considered. Then the proposed technique is applied to construction of spline curves on the smooth manifolds by action of Lie groups. It should be noted that the presented technique enables generation of $C^{n}$ continuous spline curves which are constructed locally. This feature makes it possible to use the proposed technique in on-line geometric applications. The presentation of the material is illustrated by figures which show examples of the proposed polynomials and spline curves constructed by means of the proposed approach on the sphere $S^{2}$. These illustrations confirm validity of the proposed technique.

\section{Appendix. Auxiliary binomial identities and formulas for higher-order derivatives}

In order to compute higher-order derivatives of the polynomials used for blending parametric curves in a Lie group the following two identities with binomial coefficients are used:

$$
\sum_{i=0}^{m}(-1)^{i}\left(\begin{array}{c}
m \\
i
\end{array}\right)=0, \quad \sum_{i=0}^{m}(-1)^{i}\left(\begin{array}{c}
m \\
i
\end{array}\right) i=0 .
$$

The binomial identities can be proved using Newton's binomial formula

$$
(a+b)^{m}=\sum_{i=0}^{m}\left(\begin{array}{c}
m \\
i
\end{array}\right) a^{m} b^{m-i}=a^{m}+\left(\begin{array}{c}
m \\
1
\end{array}\right) a^{m-1} b+\left(\begin{array}{c}
m \\
2
\end{array}\right) a^{m-2} b^{2}+\ldots+\left(\begin{array}{c}
m \\
m-1
\end{array}\right) a b^{m-1}+b^{m} .
$$

In order to prove the first identity it is sufficient to expand the sum

$$
(1-1)^{m}=\sum_{i=0}^{m}(-1)^{i}\left(\begin{array}{c}
m \\
i
\end{array}\right)=0
$$

using Newton's binomial formula. The second of the identities is proved using the following two relations between binomial coefficients:

$$
\left(\begin{array}{c}
m \\
i
\end{array}\right)=\left(\begin{array}{c}
m \\
m-i
\end{array}\right), \quad\left(\begin{array}{c}
m \\
i
\end{array}\right)=\frac{m}{i}\left(\begin{array}{c}
m-1 \\
i-1
\end{array}\right)
$$

It is obtained that

$$
\begin{aligned}
\sum_{i=0}^{m}(-1)^{i}\left(\begin{array}{c}
m \\
i
\end{array}\right) i & =\left(\begin{array}{c}
m \\
0
\end{array}\right) 0-\left(\begin{array}{c}
m \\
1
\end{array}\right) 1+\left(\begin{array}{c}
m \\
2
\end{array}\right) 2-\ldots-\left(\begin{array}{c}
m \\
m-1
\end{array}\right)(m-1)+\left(\begin{array}{c}
m \\
m
\end{array}\right) m \\
& =\left(\begin{array}{c}
m \\
0
\end{array}\right) \frac{m}{2}-\left(\begin{array}{c}
m \\
1
\end{array}\right) \frac{m}{2}+\left(\begin{array}{c}
m \\
2
\end{array}\right) \frac{m}{2}-\ldots-\left(\begin{array}{c}
m \\
m-1
\end{array}\right) \frac{m}{2}+\left(\begin{array}{c}
m \\
m
\end{array}\right) \frac{m}{2} \\
& =\frac{m}{2} \sum_{i=0}^{m}(-1)^{i}\left(\begin{array}{c}
m \\
i
\end{array}\right)=0
\end{aligned}
$$

for all even $m \in N$. And analogously

$$
\begin{aligned}
\sum_{i=0}^{m}(-1)^{i}\left(\begin{array}{c}
m \\
i
\end{array}\right) i & =\left(\begin{array}{c}
m \\
0
\end{array}\right) 0-\left(\begin{array}{c}
m \\
1
\end{array}\right) 1+\left(\begin{array}{c}
m \\
2
\end{array}\right) 2-\ldots+\left(\begin{array}{c}
m \\
m-1
\end{array}\right)(m-1)-\left(\begin{array}{c}
m \\
m
\end{array}\right) m \\
& =-\left(\begin{array}{c}
m \\
0
\end{array}\right) \frac{m}{2}+\left(\begin{array}{c}
m \\
1
\end{array}\right) \frac{m-2}{2}-\left(\begin{array}{c}
m \\
2
\end{array}\right) \frac{m-4}{2}+\ldots+\left(\begin{array}{c}
m \\
m-1
\end{array}\right) \frac{m-2}{2}-\left(\begin{array}{c}
m \\
m
\end{array}\right) \frac{m}{2}
\end{aligned}
$$




$$
\begin{aligned}
= & -\left(\begin{array}{c}
m \\
0
\end{array}\right) \frac{m}{2}+\left(\begin{array}{c}
m \\
1
\end{array}\right) \frac{m}{2}-\left(\begin{array}{c}
m \\
2
\end{array}\right) \frac{m}{2}+\ldots+\left(\begin{array}{c}
m \\
m-1
\end{array}\right) \frac{m}{2}-\left(\begin{array}{c}
m \\
m
\end{array}\right) \frac{m}{2} \\
& -\left(\begin{array}{c}
m \\
1
\end{array}\right) 1+\left(\begin{array}{c}
m \\
2
\end{array}\right) 2-\left(\begin{array}{c}
m \\
3
\end{array}\right) 3+\ldots+\left(\begin{array}{c}
m \\
m-2
\end{array}\right) 2-\left(\begin{array}{c}
m \\
m-1
\end{array}\right) 1 \\
= & -\frac{m}{2} \sum_{i=0}^{m}(-1)^{i}\left(\begin{array}{c}
m \\
i
\end{array}\right)-\left(\begin{array}{c}
m-1 \\
0
\end{array}\right) m+\left(\begin{array}{c}
m-1 \\
1
\end{array}\right) m \\
& -\left(\begin{array}{c}
m-1 \\
2
\end{array}\right) m+\ldots+\left(\begin{array}{c}
m-1 \\
m-2
\end{array}\right) m-\left(\begin{array}{c}
m-1 \\
m-1
\end{array}\right) m \\
= & -\frac{m}{2} \sum_{i=0}^{m}(-1)^{i}\left(\begin{array}{c}
m \\
i
\end{array}\right)-m \sum_{i=0}^{m-1}(-1)^{i}\left(\begin{array}{c}
m-1 \\
i
\end{array}\right)=0
\end{aligned}
$$

for all odd $m \in N$. Thus the second identity with binomial coefficients is also proved.

In order to compute higher-order derivatives of blending parametric curves it is necessary to use two expressions for higher derivatives of composite functions and matrix products.

It is known from the real analysis that a higher-order derivative of the composite function $f(g(u))$ can be determined using the chain rule as follows:

$$
(f(g(u)))^{(n)}=\sum \frac{n !}{k_{1} ! k_{2} ! \ldots k_{n} !} f^{(k)}(g(u))\left(\frac{g^{\prime}(u)}{1 !}\right)^{k_{1}}\left(\frac{g^{\prime \prime}(u)}{2 !}\right)^{k_{2}} \ldots\left(\frac{g^{(n)}(u)}{n !}\right)^{k_{n}}
$$

where the sum is over all nonnegative integer solutions of the Diophantine equation

$$
k_{1}+2 k_{2}+\ldots+n k_{n}=n
$$

and

$$
k=k_{1}+k_{2}+\ldots+k_{n} .
$$

For our purposes it is sufficient to note that the tuple

$$
\left(k_{1}, k_{2}, \ldots, k_{n}\right)=(n, 0, \ldots 0)
$$

is always a solution of the Diophantine equation and all other solutions imply that corresponding terms of the sum contain higher-order derivatives of the function $g(u)$.

Let $f(u)$ and $g(u)$ be two square matrices of the same order depending on the real parameter $u$. It is known from matrix calculus that a higher-order derivative of the matrix product $f(u) g(u)$ can be determined as follows:

$$
(f(u) g(u))^{(n)}=\sum_{k=0}^{n}\left(\begin{array}{l}
n \\
k
\end{array}\right) f^{(n-k)}(u) g^{(k)}(u) .
$$

\section{References}

1. M. Camarinha, F. Silva Leite and P. Crouch, 'Splines of class $C^{k}$ on non-euclidean spaces', Comput. Aided Geom. Design 12 (1995) 399-410.

2. P. Crouch, G. Kun and F. Silva Leite, 'The De Casteljau algorithm on Lie groups and spheres', J. Dyn. Control Syst. 5 (1999) 397-429.

3. P. Crouch and F. Silva Leite, 'The dynamic interpolation problem: on Riemannian manifolds, Lie groups and symmetric spaces', J. Dyn. Control Syst. 1 (1995) 177-202.

4. S. Flöry and M. Hofer, 'Constrained curve fitting on manifolds', Comput. Aided Des. 40 (2008) $25-34$.

5. M. Hofer and H. Pottmann, 'Energy-minimizing splines in manifolds', ACM Trans. Graph. 23 (2004) $284-293$.

6. J. Jakubiak, F. Silva Leite and R. C. Rodrigues, 'A two-step algorithm of smooth spline generation on Riemannian manifolds', J. Comput. Appl. Math. 194 (2006) 177-191. 
7. A. Marthinsen, 'Interpolation in Lie groups', SIAM J. Numer. Anal. 37 (1999) 269-285.

8. L. Noakes, G. Heinzinger and B. Paden, 'Cubic splines on curved spaces', IMA J. Math. Control Appl. 6 (1989) 465-473.

9. F. PARK and B. RAvani, 'Bézier curves on Riemannian manifolds and Lie groups with kinematic applications', ASME J. Mech. Des. 117 (1995) 36-40.

10. I. Pesenson, 'Variational splines on Riemannian manifolds with applications to integral geometry', Adv. Appl. Math. 33 (2004) 548-572.

11. A. P. Pobegailo, ' $C$ n interpolation on smooth manifolds with one-parameter transformations', Comput. Aided Des. 28 (1996) 973-979.

12. T. Popiel and L. NoAkes, 'Bézier curves and $C^{2}$ interpolation in Riemannian manifolds', J. Approx. Theory 148 (2007) 111-127.

13. R. C. Rodrigues, F. Silva Leite and J. Jakubiak, 'A new geometric algorithm to generate smooth interpolating curves on Riemannian manifolds', LMS J. Comput. Math. 8 (2005) 251-266.

14. D. F. Rogers and J. A. Adams, Mathematical elements for computer graphics (McGraw-Hill, New York, 1990).

15. F. W. WARner, Foundations of smooth manifolds and Lie groups (Springer, New York, 1983).

\author{
A. P. Pobegailo \\ Belarusian State University \\ Faculty of Applied Mathematics and \\ Computer Science \\ Pr. Nezavisimosti 4 \\ 220030, Minsk \\ Belarus \\ pobegailo@bsu.by
}

